\title{
Structural and optical characterization of thick and thin polycrystalline diamond films deposited by microwave plasma activated CVD
}

\author{
S K PRADHAN*, B SATPATI, B P BAG and T SHARDA ${ }^{\dagger}$ \\ Institute of Minerals and Materials Technology, Bhubaneswar 751 013, India \\ ${ }^{\dagger}$ Seki Technotron Corporation, 5-6-30 Kiba, Koto-ku, Tokyo 135 042, Japan
}

MS received 10 February 2009

\begin{abstract}
Preliminary results of growth of thin diamond film in a recently installed $3 \mathrm{~kW}$ capacity microwave plasma activated CVD (MW-PACVD) system are being reported. The films were deposited on Si (100) substrate at $850^{\circ} \mathrm{C}$ using methane and hydrogen mixture at $1.5 \mathrm{~kW}$ MW power. The grown polycrystalline films were characterized by micro-Raman, transmission electron microscope (TEM), spectrophotometer and atomic force microscope (AFM). The results were compared with that of a thicker diamond film grown elsewhere in a same make MWPACVD system at relatively higher power densities. The presence of a sharp Raman peak at $1332 \mathrm{~cm}^{-1} \mathrm{confirmed}^{-}$ the growth of diamond, and transmission spectra showed typical diamond film characteristics in both the samples. Typical twin bands and also a quintuplet twinned crystal were observed in TEM, further it was found that the twinned region in thin sample composed of very fine platelet like structure.
\end{abstract}

Keywords. Diamond film; plasma CVD; defects; optical properties.

\section{Introduction}

Diamond is one of the most sought after material not only for its gemmological properties but also for its technical qualities. It has combinations of properties which are unsurpassed by any other material: highest hardness and thermal conductivity, low thermal expansion and high thermal shock resistance, excellent chemical and radiation resistance, broad spectrum optical transparency, low coefficient of friction, high electrical resistivity etc (Asmussen and Reinhard 2002). Also it is a very promising high power semiconductor material as it is a wide-bandgap semiconductor with high electron and hole mobility even at high doping levels (Wort and Balmer 2008).

There is much interest in the research and industrial community to grow diamond films on large area so that it can be used in microelectronics and other engineering applications. In the last 25 years, the growth of pure diamond films by vapour deposition has come a long way, from early reports of growth of diamond by Eversole (1962) to the current situation of growth of thick polycrystalline, highly oriented (Han et al 2000) homoepitaxial and heteroepitaxial films (Jiang et al 2000; Okushi 2001; Bogdan et al 2005; Man et al 2006). Out of several vapour deposition methods, two are dominating the development: MW-PACVD and hot filament CVD (HF-CVD). In general, the MW grown films are considered

\footnotetext{
*Author for correspondence (skpradhan@immt.res.in)
}

superior in quality than the other. In HF-CVD there is more chance for incorporation of metal impurities coming from the heated filament, and also depending on the filament material there is restriction on the type of gas that can be fed (i.e. oxidizing or corrosive species may not be suitable).

To grow high quality diamond films for applications in $\mathrm{X}$-ray and laser optics, thermal management systems, etc we started investigating structural and optical properties of thin and thick diamond films deposited in a commercial MWPACVD reactor. Some preliminary results obtained by characterizing these films by high resolution TEM, microRaman and AFM are reported in this article.

\section{Experimental}

Diamond films were grown using $1 \% \mathrm{CH}_{4}$ (in balance $\mathrm{H}_{2}$ ) in a MW-PACVD Model AX5230S (made by Seki Technotron Corp., Japan) at $850^{\circ} \mathrm{C}$ and $1.5 \mathrm{~kW}$ microwave power on silicon (100) substrates. The thicker diamond film with which the structural and optical characteristics of the thin diamond film are compared was grown elsewhere in a similar MW-PACVD system and at the same conditions but using microwave power of $4 \mathrm{~kW}$. Films were characterized by micro Raman (Renishaw inVia) with $514 \mathrm{~nm}$ green laser beam, TEM (FEI, TECNAI G ${ }^{2} 20$, TWIN) operating at $200 \mathrm{kV}$, AFM (DME Dualscope) and spectrophotometer (Hitachi). TEM samples were prepared by mechanical polishing and ion milling. A $3 \mathrm{~mm}$ disc sample was ultrasonically cut from an as-grown film. This sample was then mounted on a specimen lapping holder with precise scales 


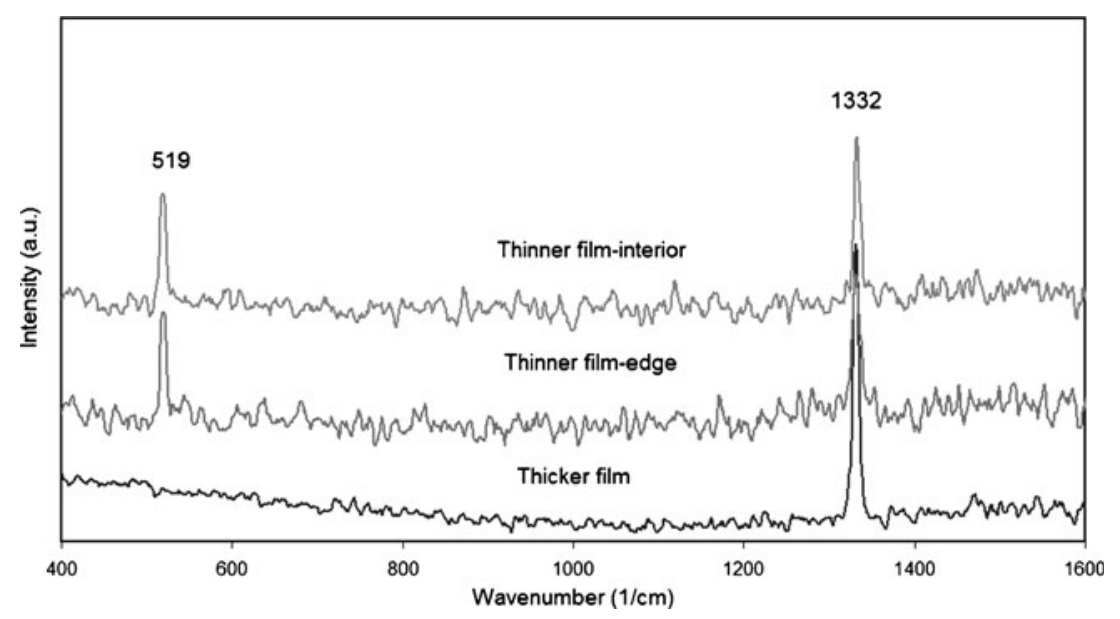

Figure 1. Raman spectra of grown diamond films.

and mechanically polished to a thickness of $100 \mu \mathrm{m}$ from the substrate side using 600, 800 and 1200 grit silicon carbide paper and finally with fine grade emery paper. It was further thinned to $20 \mu \mathrm{m}$ in the centre of the sample with a dimpling grinder to facilitate subsequent ion thinning. Then it was put into an ion milling machine for final thinning. In the ion miller, argon ion beams were accelerated with a voltage of 3-4 kV and a current of $20 \mu \mathrm{A}$, bombarding the sample at a small angle $\left(4^{\circ}\right)$. The samples were thus ion milled until perforation occurred. The area around the perforated hole was normally thin enough for TEM observations. During the final stage of ion milling when perforation was about to occur, a reduced voltage and current was applied to the argon ion beam to avoid possible damage to the sample surface by ion bombardment (e.g. graphitization or amorphization).

\section{Results and discussion}

The Raman spectra of the grown films are shown in figure 1 ; as seen clearly, there is a sharp feature at $1332 \mathrm{~cm}^{-1}$ for all the three spectra (two spectra from the thinner films and one for the thick film), which is an unambiguous signature of diamond, and the other sharp feature near $520 \mathrm{~cm}^{-1}$ in thin film appeared from the Si substrate. Sharp feature at $1332 \mathrm{~cm}^{-1}$ is the signature peak of diamond and absence of graphitic bands, respectively at $1350 \mathrm{~cm}^{-1}$ and $1580 \mathrm{~cm}^{-1}$, which indicate phase pure diamond film. To check the structural uniformity, spectra were obtained across the two inch area of the films. As shown in the figure, the Raman structure of the film from edge to centre of the film does not vary, thus suggesting structural uniformity across entire area of the film.

CVD grown diamond films usually have large number of structural defects such as twins, stacking faults and dislocations (Shechtman et al 1993; Jiang and Jia 2002; Erni

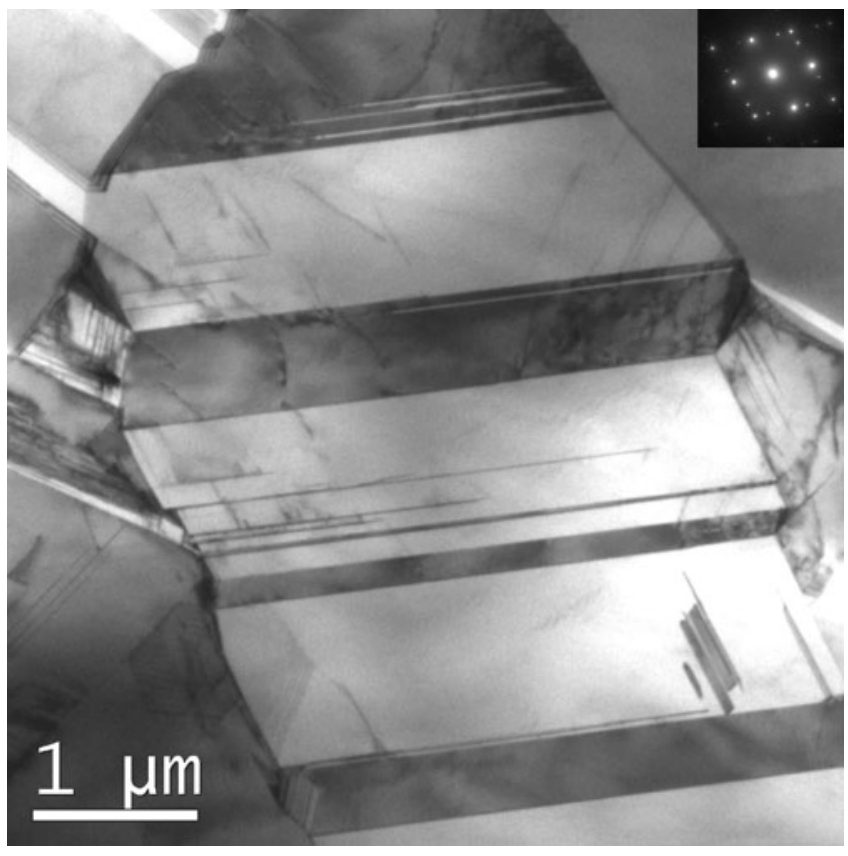

Figure 2. TEM image of thick diamond film from a twinned region; inset shows corresponding SAD pattern.

et al 2006). TEM image of the thicker film is shown in figure 2 with selected area diffraction (SAD) pattern in the inset. Figure 3 shows TEM images and SAD of thinner film.

Twining in diamond crystal is very common. There are exotic twin configurations including quintuplet twins (Shechtman et al 1993; Sawada and Ichinose 2005). As shown in figure 2, extensive twin bands, observed in the thicker films and extending through the grains, appear like a three-dimensional staircase. Twin boundaries are often found to consist of flat segments in particular orientations. The firstorder twin boundary in $f c c$ crystals like diamond tends to 

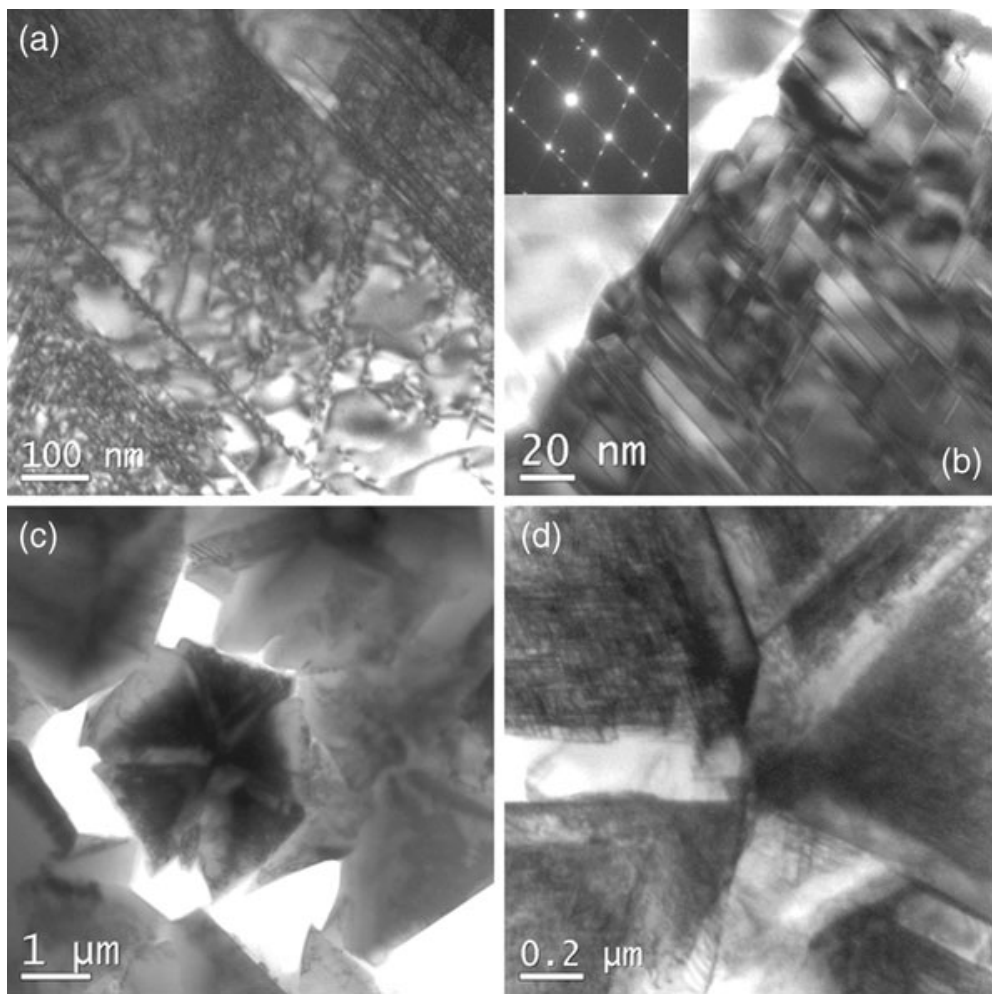

Figure 3. TEM and SAD patterns of thinner film: (a) twinning bands with fine platelet in it, (b) magnified view of platelets with SAD (inset), (c) quintuplet twinned crystal and (d) magnified view of quintuplet showing fine platelets.

facet parallel to the common $\{111\}$ plane, as shown in the inset of figure 2. This means that if the specimen is oriented so that the common planes are nearly parallel to the beam, the common $\{111\}$ reflection will be excited. However, in case of the thinner film, as shown in figure 3(a), a fewer number of twin bands were observed. A magnified image from figure 3(a) is shown in figure 3(b), which clearly reveals the presence of very thin plate-like precipitates; these plate-like structures are further verified by the appearance of long streaks in diffraction pattern, as shown in the inset of figure 3(b). The nature of multiply twinned particles (MTPs) in the films are also studied using TEM. In a separate area in thin film, a crystal having a five-fold MTP configuration was observed as shown in figures $3 \mathrm{c}$ and $\mathrm{d}$. In figure $3 \mathrm{c}$, a bright field micrograph of a five-fold MTP shows five growth sectors comprising of tetrahedron bounded by $\{111\}$ planes sharing a common $\left[\begin{array}{lll}1 & 1 & 0\end{array}\right]$ axis and twinned relative to each other. The electron beam is parallel to a $\left[\begin{array}{lll}1 & 1 & 0\end{array}\right]$ direction. On close observation, in the quintuplet twinned regions the structure was composed completely of small platelets. The presence of platelets in the crystal was further revealed by the SAD pattern that showed streaks connecting the spots (similar to figure 3 (b) inset). The twin formation during crystal growth can be explained by the model proposed by Butler and Oleynik (2008). According to their model, formation of an isomerizing three-atom bridge with equal chance for formation of a stable fouratom nucleating island leads to twin growth, depending on the local growth conditions (arrival of $\mathrm{C}$ atoms or radicals), the three-atom bridge can grow into a four-atom island with either normal or twin orientation relative to the previous layer.

AFM images in figure 4 show the film morphology and crystal size. From the images, sizes of the crystal are about 3-5 $\mu \mathrm{m}$ and 20-25 $\mu \mathrm{m}$, respectively for the thin and thick films. The measured root mean square roughness value is $180 \mathrm{~nm}$ for the thin sample and $690 \mathrm{~nm}$ for thick sample.

Transmission spectra of diamond films in the UV-visible range are shown in figure 5. Both the films have more than $80 \%$ transmittance, which means that these are of optical grade suitable for optical window applications in lasers and other critical applications. However, as measured by the AFM, thicker film is comparatively much rougher than the thinner film. Obviously, larger the grains rougher the films are, which does not allow them to be used for optical window or as optical and tribological coatings (Sharda and Bhattacharya 2003; Quadrini et al 2008). However, polishing increases the cost substantially and thus, as grown fine-grained nanocrystalline diamond films will be better suitable for optical window and/or coating applications. 

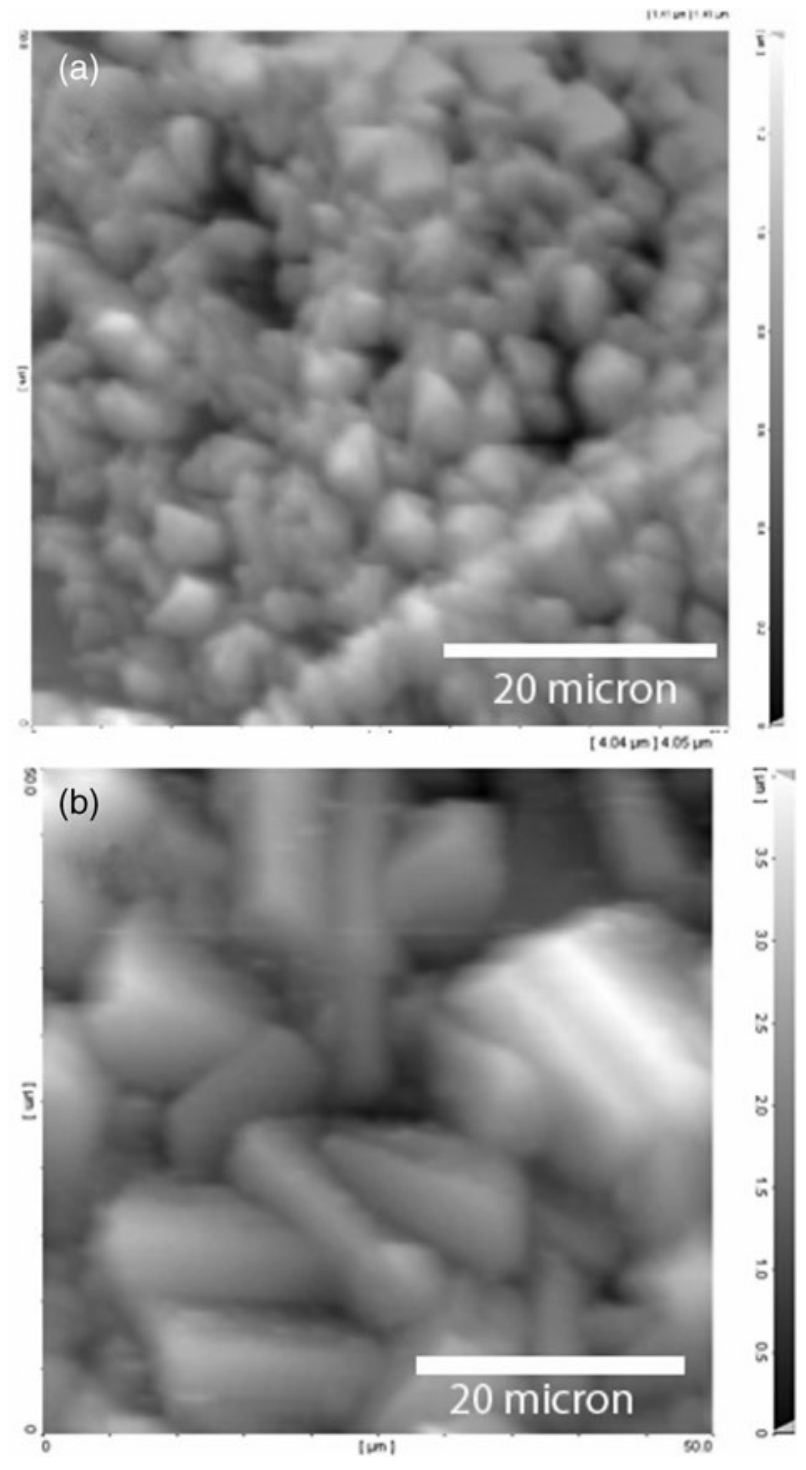

Figure 4. AFM images of diamond films: (a) thin film and (b) thick film.

\section{Conclusions}

The Raman spectra of diamond films clearly showed that high quality pure diamond films grew on $\mathrm{Si}(100)$ substrates. It was observed that thicker film had large number of twin bands which run through the crystals, whereas the thinner film had lesser number of twin bands which composed of fine platelets. SAD pattern from the thinner film distinctly showed streaks connecting the spots while a clear SAD pattern was observed in the case of thicker film. In spite of the large differences in microstructural features in these films, both films showed high transmittance in the UV-visible range. However, due to rougher morphology thinner or finegrained films would be better suitable for optical window and coating applications.

\section{Acknowledgement}

The authors would like to thank the Director, IMMT Bhubaneswar, for support and encouragement.

\section{References}

Asmussen J and Reinhard D K 2002 Diamond films handbook (eds) J Asmussen and D K Reinhard (New York: Marcel Dekker), Ch. 1 Bogdan G, Nesládek M, D’Haen J, Maes J, Moshchalkov V V, Haenen K and D'Olieslaeger M 2005 Phys. Status Solidi A202 2066

Butler J and Oleynik I 2008 Philos. Trans. R. Soc. A366 295

Erni R et al 2006 Microsc. Microanal. 12492

Eversole W G 1962 Synthesis of diamond, US Patents No. 3030187

Han S K, McClure M T, Wolden C A, Vlahovic B, Soldi A and Sitar S 2000 Diamond Relat. Mater. 91008

Jiang J, Fryda M and Jia C L 2000 Diamond Relat. Mater. 91640

Jiang X and Jia C L 2002 Appl. Phys. Lett. 802269

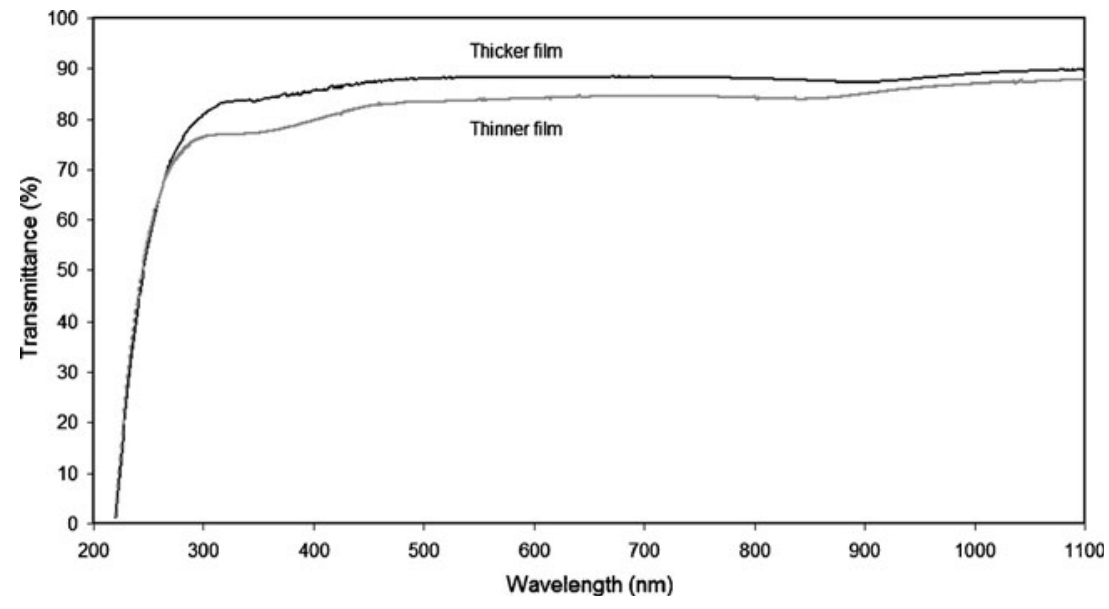

Figure 5. Transmission spectra of diamond films in UV-visible range. 
Man W, Wang J, Wang C, Ma Z, Wang S and Xiong L 2006 Plasma Sci. Technol. 8329

Okushi H 2001 Diamond Relat. Mater. 10281

Quadrini F, Tagliaferri V and Polini R 2008 Mach. Sci. Technol. 12 390

Sawada H and Ichinose H 2005 Diamond Relat. Mater. 14109
Sharda T and Bhattacharya S 2003 Encyclopedia of nanoscience and nanotechnology (ed) H S Nalwa (California: American Scientific Publishers) Vol. 2, p. 337

Shechtman D, Hutchison J L, Robins L H, Farabaugh E N and Feldman A 1993 J. Mater. Res. 8473

Wort C J H and Balmer R S 2008 Mater. Today 1122 